\title{
Regeneration der Kiefer bei der Eidechse Lacerta agilis.
}

\author{
Von \\ Isaak Werber. \\ (Aus der Biologischen Versuchsanstalt in Wien.)
}

Mit 4 Figuren im Text.

Eingegangen am 7. Dezember 1904.

In dem großen Tatsachenmaterial, das uns über die regenerativen Potenzen der Tiere vorliegt, sind mehrere Lücken zu finden, die nach den bisherigen Beobachtungen und den Ergebnissen der bis nun vorgenommenen Versuche zu schließen - in vielen Fällen leicht auszufüllen wären. Wenn nämlich »die regenerativen Potenzen um so mehr abnehmen, je weiter sich das betreffende Tier vom einfachen Baue der Einzelligen entfernt" (Fraisse 1885, Nussbaum 1886, BarFurth in den Erg., Loeb 1895/6, Przibram 1899), müßte jeder Schluß aus dem Vorhandensein einer gewissen Regenerationsfähigkeit bei einem gewissen Tiere auf die Annahme derselben bei einem im System niederer Stehenden als vollkommen berechtigt angesehen werden, insofern das betreffende Glied nicht etwa eine besondere Spezialisierung aufweist. Da jedoch dagegen Einwände durch LESsona, Weisuaxy und Bordage erhoben wurden, die die Regeneration für ein bestimmtes Glied auf seine Verlnstwahrscheinlichkeit zurückführen, dürfen wir uns mit solchen Schltissen allein nicht zufrieden geben; wir sollen es vielmehr nicht unterlassen, die erwähnten Lücken in solchen Fällen wenigstens, wo dies nicht auf unüberwindbare Schwierigkeiten stößt, nach Möglichkeit auszufüllen. Zudem ist es in vielen Fällen nicht allein die zu konstatierende Tatsache der Regeneration, um derentwillen die Versuche sich lohnen, es sind auch die Ergebnisse der Begleiterscheinungen, die oft von viel größerem Interesse und Bedeutung sind, als die Tatsachen einer stattfindenden Regeneration an und für sich, nicht zu unterschätzen. 
Die in den letzten Jahren bekannt gewordenen Tatsachen der Schnabelregeneration bei den Vögeln legten es nahe durch Versuche zu ermitteln, ob homologe Körperteile bei den den Vögeln nahestehenden Reptilien zu regenerieren vermögen. Zu den Versuchen, die zunächst an Lacerta agilis vorgenommen wurden, wurden Tiere beiderlei Geschlechter und verschiedenen Alters verwendet und es erwies sich, daß weder Geschlecht noch Alter des Tieres von bedeutendem Einflusse auf die Regeneration sind. Von Einfluß dürfte da nur die Art der vorgenommenen Operation sein, nämlich welche Partien des Kiefers entfernt werden. Ich nahm die Operation an einigen Exemplaren nur am Oberkiefer vor, an allen übrigen an beiden Kiefern, weil ich etwaige Unterschiede in der Qualität und der Zeitdauer der allenfallsigen Regeneration erwartete, je nachdem der eine oder beide Kiefer verletzt worden waren, was jedoch nicht der Fall war. Die Operation wurde mittels Schere ausgefuhrt, die nach jedem Schnitt durch die Flamme gezogen wurde, um eine etwaige Infektion zu vermeiden. Ich entfernte an allen Exemplaren immer dieselbe Partie der Kiefer, nämlich das Intermaxillare am Oberkiefer und das Symphysiale am Unterkiefer. Dabei konnte ich aber nicht darauf achten, die genannten Partien scharf nach ihren Abgrenzungen herauszuschneiden, da die Operation möglichst rasch geschehen mußte, um eine starke Blutung zu verhüten. Das Symphysiale (bzw. Intermaxillare) wurde also mit zwei geradlinigen Schnitten entfernt, so, daß das entfernte Stück wie ein mehr oder minder reguläres Dreieck aussah. Die Tiere wurden vor der Operation mit Äther betäubt, nach der Operation in eine Glaswanne gebracht, und nachdem sie sich aus dem Betäubungszustand erholt hatten, in Käfige untergebracht. Die der Sonne zugewendeten Käfige aus Glasscheiben in Eisen- oder Holzrahmen (eine Wand war aus einem Drahtnetz, damit die Luft im Käfig frisch erhalten bleibe) waren mit einer Schicht von Kies, der mit Moos bedeckt wurde, ausgebettet, enthielten eine oder mehrere Pflanzen und einige Steine, unter welche sich diese Tiere sehr gern verkriechen. Die Zimmertemperatur betrug $+25^{\circ} \mathrm{C}$. Die Tiere wurden mit Larven vom Mehlkäfer oder mit Hausfliegen gefittert. Ziemlich oft wurden die Käfige gereinigt, um den Ausbruch einer Seuche zu verhüten.

Der Verlauf des Regenerationsprozesses ist aus folgendem Protokolle zu ersehen: 
Serie I.

A. Operation.

6. V. 1904. An 4 Exemplaren am Oberkiefer das Intermaxillare herausgeschnitten. Bei einem Tier ist der Schnitt zufälligerweise etwas zu tief gefübrt worden, so, daß mit dem Schnitt auch das linke Nasenloch entfernt wurde.

B. Beobachtungen nach der Operation.

7. V. 1904. Ein Wundschorf hat sich gebildet und die Wunde ist bereits geschlossen.

16. V. 1904. Die durch Entfernung des Intermaxillare entstandene Licke im Kiefer wird immer kleiner, die Winkelarme der Lieke kommen immer näher aneinander.

21. V. 1904. Der Winkel an der operierten Stelle ist schon sehr klein; die Liicke am Kiefer verschwindet.

26. V. 1904. Häutung eines Exemplars. Die durch die Operation entstandene Lücke ist gänzlich verschwunden, da das Intermaxillare bereits regeneriert ist. Die Regeneration ist noch nicht vollständig; es fehlt noch die Beschuppung. Das Tier wurde in Alkohol konserviert.

20. VI. 1904. An einem Exemplar, dem bei der Operation das linke Nasenloch weggeschnitten wurde, ist an der rechten Seite das Regenerat fast vollständig hervorgetreten, an der linken Seite dagegen nur schwach, aber die Bildung des Nasenloches ist deutlich wahrzunehmen.

9. VIII. 1904. Ein Exemplar mit vollständigem Regenerat. An Stelle des der entfernten Partie zukommenden einen Schildes sind viele kleinere Schuppen (Granulaschuppen) wahrzunehmen.

\section{Serie II.}

A. Operation.

7. V. 1904. An 12 Exemplaren am Unterkiefer das Symphysiale und am Oberkiefer das Intermaxillare herausgeschnitten.

B. Beobachtungen nach der Operation.

8. V. 1904. Wundschorf und Verschluß der Wunde.

16. V. 1904. Die durch Entfernung des Intermaxillare und Symphysiale in den Kiefern entstandenen Licken werden immer kleiner, die Winkelarme der Litcke im Intermaxillare kommen immer näher aneinander; der Unterkiefer spitzt sich zu und wächst nach. 
21. V. 1904. Die Lücke im Oberkiefer ist schon sehr klein, der Unterkiefer ist schon fast gänzlich nachgewachsen.

30. V. 1904. An einem kleinen (etwa $6 \mathrm{~cm}$ langen) Exemplar ein Regenerat am Oberkiefer und am Unterkiefer konstatiert. An dem Regenerat des Unterkiefers, der seine normale Länge noch nicht erreicht hat, kann man mittels Lupe schon die regenerierten Zähnchen sehen. Das Tier wurde behufs Konstatierung, ob der Unterkiefer seine normale Länge noch erreichen wird, isoliert.

14. VI. 1904. Bei dem am 30. V. 1904 isolierten Exemplar ist am Regenerat des Oberkiefers die Beschuppung in Ausbildung begriffen. Dieselbe ist von der primären ganz verschieden; an Stelle des dem Intermaxillare zukommenden einen Schildes (Rostrale) sind mehrere kleinere Körnerschuppen zu sehen. Der Unterkiefer ist bedeutend gewachsen.

20. VI. 1904. An einem trächtigen Weibchen (das zur Zeit der Operation noch nicht trächtig gewesen war) wurde konstatiert: Unterkiefer vollständig regeneriert, sieht genau so aus, wie der normale; Oberkiefer fast vollständig regeneriert mit in Ausbildung begriffenen Granulaschuppen an Stelle des Rostrale bedeckt.

4. VIII. 1904. Vollständige Regenerate an 3 Exemplaren. Die Beschuppung ist an beiden Kiefern von der primären ganz verschieden: Granulaschuppen. Alle 3 Exemplare in Alkohol konserviert.

\section{Serie III.}

\section{A. Operation.}

9. V. 1904. An 4 Exemplaren am Unterkiefer das Symphysiale und am Oberkiefer das Intermaxillare herausgeschnitten.

B. Beobachtungen nach der Operation.

10. V. 1904. Wandschorf und Wundverschluß.

16. V. 1904. Die durch Entfernung des Intermaxillare und Symphysiale in den Kiefern entstandenen Lücken werden immer kleiner, die Winkelarme der Lücke im Intermaxillare kommen immer näher aneinander; der Unterkiefer spitzt sich zu und wächst nach.

21. V. 1904. Die Liucke am Oberkiefer ist nunmehr sehr klein; der Unterkiefer ist bedeutend nachgewachsen.

4. VIII. 1904. Ein - Exemplar hat Unterkiefer und Oberkiefer vollständig regeneriert. An beiden Kiefern sind Granulaschuppen wahrzunehmen. Das Tier wurde in Alkohol konserviert.

7. VIII. 1904. Desgl. 
(Einige - etwa 5-6 - Exemplare aus allen Serien sind während der Versuchsperiode gestorben.)

Die Operation verursachte bei den Tieren eine sehr geringe Blutung aus der Wundstelle, die mit Eisenchlorid-Watte gehemmt wurde. Sehr junge Exemplare bluteten gar nicht. Der Verschluß der Wundränder erfolgte rasch, war immer schon einen Tag nach der Operation zu bemerken. Es bildete sich ein Wundschorf, nach dessen Abwerfen die durch die Operation im Kiefer entstandene Lücke immer kleiner wurde, die Winkelarme dieser lüucke rückten immer näher aneinander, die Lücke füllte sich, bis sie endlich gänzlich schwand und an ihrer Stelle ein Regenerat von der urspriunglichen Größe des entfernten Kieferteils zu konstatieren war. Daß auch die etwas tiefer liegenden Partien zu regenerieren vermögen, ist aus der Tatsache ersichtbar, daß bei einem Exemplar, bei dem der Schnitt zufällig etwas tiefer geführt wurde, so, daß auch das linke Nasenloch weggeschnitten wurde, dasselbe wieder neu gebildet und ein vollständiges Regenerat erzielt wurde.

Was die histologische Beschaffenheit des Regenerats betrifft, muß vorläufig nur hervorgehoben werden, daß an Querschnitten der regenerierten Kieferteile anstatt des Knochengewebes typisches Knorpelgewebe zu konstatieren ist. Wegen ungünstiger Konservierung der untersuchten Objekte waren die Schnittpräparate zu einer genaueren Untersuchung ungeeignet. In einer demnächst folgenden ausführlichen Arbeit über die Regenerationsverhältnisse bei den Reptilien, mit der ich jetzt beschäftigt bin, soll diesem Umstande Rechnung getragen werden. Dieses interessante Verhältnis, daß Knochengewebe des Kiefers infolge Regeneration durch Knorpelgewebe ersetzt wird, und die lange bekannte Tatsache, daß die regenerierten Schwänze der Eidechsen keine Wirbel, sondern einen Knorpelstab enthalten Perrault, 1688), dürften etwa zu einer Vermutung berechtigen, daß Knochensubstanz bei den Reptilien (oder zu mindestens bei den Eidechsen) nicht mehr als solche regeneriert wird. Doch scheint es mir nicht ganz ausgeschlossen, daß das Knorpelgewebe des regenerierten Schwanzes (und möglicherweise auch das der regenerierten Kieferteile) mit zunehmendem Alter des Regenerats einer Ossifikation unterliegt - ein Umstand, der auch noch berücksichtigt werden soll.

Von großem Interesse ist noch die Beschuppung des Regenerats. Das Intermaxillare ist bekanntlich bei den Lacertiden mit einem Schilde, nämlich dem Rostrale, bedeckt (Fig. 1). Ebenso kommt am Unterkiefer dem Symphysiale nur ein einziges Schild zu (Fig. 3). 
Von dieser primären Beschilderung weicht die des Regenerats gänzlich ab (Fig. 2, 4). Anstatt des einen normalen Schildes weist das Regenerat bei manchen Exemplaren einige kleinere und bei andern sehr viele ganz kleine Schuppen (Granulaschuppen) auf. Ganz ähnliche Verhältnisse sind bei der Regeneration des Schwanzes vieler

Fig. 1.

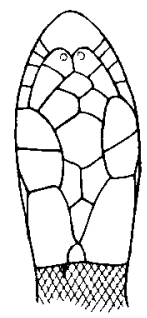

Fig. 2.

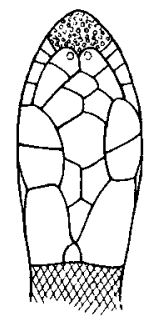

Eidechsenarten bekannt, die von Boulenger (1891), LydEKKER und Werner (1896) eingehend untersucht, zur Behauptang An]aß gaben, daß die von der normalen abweichende Schuppenbekleidung des regenerierten Schwanzes bei vielen Eidechsen auf phylogenetisch ältere Formen der betreffenden Familien zurückzuführen sei. Diese sehr wichtige Annahme kann nun auf die Beschuppung der regenerierten Kiefer erweitert werden. In dieser Annahme Fig. 3.

Fig. 4.
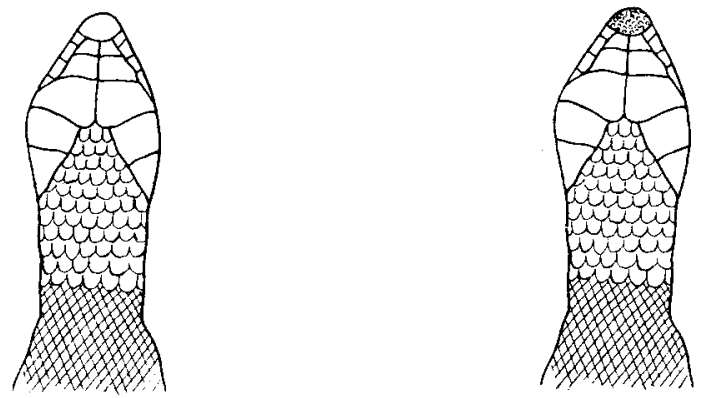

stimme ich ganz mit Werser überein, bei dem es an einer andern Stelle heißt: "Die Ursprünglichkeit der Kopfbedeckung mit kleinen Schuppen für die Eidechsen muß man als richtig anerkennen. « Die Schuppenbekleidung der regenerierten Kiefer bei den Eidechsen ist also diejenige, die Werner als die ursprüngliche treffend annimmt, wenn auch diese seine Annahme sich lediglich auf Vergleichung und nicht auf experimentelle Beweise stützt. Die von der primären abweichende Beschuppung der regenerierten Kiefer, die ich bei der 
Lacerta agilis konstatierte, ist somit die einer phylogenetisch älteren Form dieser Familie. Wir haben es also hier meiner Ansicht nach mit einem Atavismus zu tun, wie er bei der als shpotyp bezeichneten Regeneration oft hervortritt.

Wenn wir nun an die Lösung des eingangs gestellten Problems schreiten, so sehen wir, daß wir die Möglichkeit einer Regeneration der Kieferspitzen bei Eidechsen voraussagen konnten, nachdem die Regeneration analoger Körperteile bei den den Reptilien nahestehenden, jedoch höher entwickelten Vögeln bekannt geworden war, unter der Voraussetzung, daß die Regeneration eines verletzten oder verloren gegangenen Körperteils von der Organisationshöhe und dem Alter des Tieres, aber nicht von der Verlustwahrscheinlichkeit des betreffenden Körperteils abhängt. -

Versuchen wir jetzt dieselbe .Voraussetzung auch auf die Regeneration des Schnabels bei den Vögeln anzuwenden. Bezüglich dieses letzteren Falles (der Schnabelregeneration) möchte ich auf die Ausfuhrungen WeIsmanss hinweisen, gegen welche der vorliegende Versuch und meine Beobachtungen bezüglich der Schnabelregeneration beim Haushuhn, die ich weiterhin besprechen will, einen gentigenden Gegenbeweis liefern dürften. WeismanN sucht nämlich zu beweisen, daß die bekannten Fälle der Regeneration des Schnabels bei den Vögeln adaptativer Natur sind und daß somit die Regeneration beim ausgebildeten Tier nicht auf eine allgemeine Regenerationskraft zu beziehen sei. Und so finden wir in einer seiner Schriften (1899) folgende Stelle:

2.. . Das alles stimmt mit dem aufgestellten Prinzip, wonach das Regenerationsvermögen eines Tieres oder Teils durch Anpassung an die Verlnstfähigkeit und die Höhe des Verlustschadens reguliert wird. Dagegen schien bisher mit dieser Auffassung nicht vereinbar ein von KENNEL mitgeteilter, von mir schon früher hervorgehobener Fall des Storches, dem der Oberschnabel zufällig in der Mitte abgebrochen und darauf der Unterschnabel an derselben Stelle abgesägt worden war, und der beide wieder vollstïndig regenerierte.

\Daß ein Abbrechen des Schnabels bei Vögeln öfter's vorkommen sollte. konnte man damals nicht annehmen, da keine weiteren Beobachtungen darïber bekannt waren, und so bildete dieser Fall eine Schwierigkeit für die Theorie, sie schien darauf hinzudeuten, daß die Regenerationskraft eines Teils nicht auf spezieller Anpassung desselben an eine hohe Verlustmöglichkeit bei hohem biologischem Wert des Organs beruhe, sondern daß sie eine allgemeine Anpassung sei, eine Regenerationskraft des ganzen Organismus, die bis zu einem gewissen Grad ibberall in Tätigkeit treten könne, wo an dem betreffenden Tier etwas verloren geht, geschehe dies auch ganz ausnahmsweise.

- Dieser scheinbare Widerspruch gegen die Anpassungstheorie der Regeneration wird nun durch die Mitteilungen BORDAGEs beseitigt, indem er auf der 
Insel Bourbon beobachtete, daß Verletzungen des Schnabels bei Hähnen, die zu dem dort beliebten Hahnenkampf benutzt werden, häufig vorkommen und regelmäßig zur Regeneration des Schnabels führen. Er fand, daß der Schnabel der Hähne nach dem Gefecht beschädigt war, daß aber nachher eine ganz vollständige Regeneration sich vollzog. Die Beschädigungen geschehen teils durch Schnabelhiebe, teils auch, par un terrible coup de patte', erstrecken sich aber höchstens auf das Enddrittel des einen oder beider Kiefer, ,ce qui représente, pour la mandibule supérieure le prémaxillaire ou intermasculaire, os impair résultant de la soudure des intermaxillaires, et pour la mandibule inférieure, la partie triangulaire formée par la soudure des deux maxillaires, à leur extremité terminales. Diese Partien des Schnabels können vollständig abgebrochen sein, und dann erfolgt eine vollständige Regeneration, die sowohl die Knochen als die Hornbekleidung desselben neu hervorbringt....

`. . Obgleich diese Beobachtungen sich nur auf die vom Mensehen hervorgerufenen Kämpfe der Hähne beziehen, so haben sie doch eine größere Bedeutung, wie der Verfasser mit Recht andentet. Es ist bekannt, da $\beta$ bei zahlreichen Vögeln die Männchen zur Zeit der Fortpflanzung miteinander kämpfen, wobei natürlich der Schnabel die Hauptwaffe bildet. Gerade von den Störchen sagt schon BREнм, daß sie aus Eifersucht sich häufig tödliche Gefechte liefern. So wird man den KenNeLschen Fall nicht mehr als einen Widerspruch gegen die Ansicht von der adaptativen Natur der Regeneration ansehen können, und damit fällt die einzige Beobachtung, welche die Regeneration beim ausgebildeten Tier auf eine allgemeine Regenerationskraft zu beziehen Anlaß gaibe.

Wie wir nun sehen, dürfte nach der angeführten Ansicht WeIsmanss die Regenerationsfähigkeit des Schnabels bei den Vögeln auf geschlechtliche ZuchtwahI zuriekzuführen sein und müBte sonach vornehmlich dem Männchen zukommen. Diesen Schluß zog folgerichtigerweise schon WOLF:

»Die Entstehung der Schnabelregenerationsfähigkeit wird also auf geschlechtliche Zuchtwahl zurückgeführt. Nach den Prinzipien der geschlechtlichen Zuchtwahl pflegen im allgemeinen die in solchen Eifersuchtskämpfen erworbenen Eigenschaften sich auf die männlichen Individuen zu beschränken. Die Schnabelersatzdeterminanten miißten daher einen sekundären Sexnalcharakter der männlichen Störche (resp. Hühner) ${ }^{1}$ \} besitzen. Eine solche a priori wohl nicht wahrscheinliche Inferioritait des weiblichen Schnabels gegenüber dem männlichen kann aber festgestellt werden. Wir hätten also hier einmal eine selektionstheoretische Ableitung, die, wenigstens einigermaßen, einer empirischen Kontrolle zugänglich ist: man branchte nur einer Störchin (resp. Henne) ${ }^{1}$ ) den Schnabel abzusägen.*

Da ein solcher Fall bis nun nicht bekannt ist, weil derartige Versuche nicht angestellt wurden, will ich nun auf meine diesbeziiglichen Beobachtungen (Schnabelregeneration bei der Henne) hinweisen. Während meines vieljährigen Aufenthalts auf dem Lande hatte ich sehr häufig Gelegenheit gehabt zu bemerken, daß manchen besonders

1) Bemerkung des Ref. 
gefräßigen Bruthennen die Schnabelspitze (am Unterschnabel und Oberschnabel) in einer Länge von ungefähr $6-7 \mathrm{~mm}$ abgeschnitten wird, um dieselben an dem Auffressen ihrer eignen Bruteier zu verhindern. Hennen, an denen diese Operation vorgenommen wurde, mußten dann oft künstlich gefüttert werden, bis sie nach einer Zeit von 3 bis 4 Wochen die Schnabelspitzen vollständig regenerierten. Leider hatte diese Erscheinung für mich zu jener Zeit, da ich diese noch aus meinem Knabenalter stammenden Beobachtungen machte, kein weiteres Interesse und deshalb bin ich heute nicht in der Lage, die Partien des Schnabels, die regeneriert wurden, genau angeben zu können. Aber wenn nun schon einmal die Tatsache der Regeneration des Schnabels bei weiblichen Individuen des Huhns als bestimmt angesehen werden muß, so ist wohl kein Grund dazu vorhanden, um anzunehmen, daß die Regeneration sich hier auf einen kleineren Teil als beim männlichen Huhn beschränkt. Dasselbe wird nun für all die Vögelarten gelten, bei denen die Schnabelregeneration bei männlichen Individuen bekannt ist. In seinen $\gg$ Vorträgen über Descendenztheorie* kommt der genannte Forscher gelegentlich der Besprechung der Regenerationserscheinungen im Tierreiche noch einmal auf die Schnabelregeneration der Vögel zurtick. Er spricht da nochmals über die Schnabelregeneration beim Storebe und gibt wiederum der Meinung Ausdruck, daß wir es da mit einer sspeziellen Anpassung an eine hohe Verlustmöglichkeit bei hohem biologischen Wert des Organs « zu tun haben. Er zählt einige Vögelgattungen auf, bei denen die Verlustmöglichkeit des Schnabels eine hohe ist, unter andern auch den Specht (ron dem es allerdings bislang nicht bekannt ist, daß er den Schnabel regeneriere!). Nun fragt es sich jetzt: meint Weismans nur den männlichen Specht, der den Schnabel regenerieren soll? Das können wir ihm doch gar nicht zumuten, da die Lebensweise beim weiblichen Individuum ron Specht genau dieselbe ist, wie die beim männlichen: das Weibchen gebraucht den Schnabel zum Aufsuchen der Nahrung in der Rinde der Bäume ebensogut wie das Männchen. Dann ist also die Verlustmöglichkeit gewisser Schnabelpartien beim Weibchen genau dieselbe wie beim Männchen; folglich müßte auch die Regenerationsfähigkeit dieselbe sein. Wenn wir den letzteren Fall zugeben wurden, kann dann die Schnabelregenerationsfähigkeit bei diesen Vögeln absolut nicht auf geschlechtliche Zuchtwahl zurückgeführt werden. Die Tatsache, daß die Henne ebensogut den Schnabel regeneriert wie der Hahn, laßst nun die Anschaung Weismanss bezliglich jener Vögel, deren 
Männchen untereinander Kämpfe ausfechten, als ebenso ungerechtfeitigt erseheinen: es kann da weder von einer »speziellen Anpassung an hohe Verlustmöglichkeit « ${ }^{1}$ ) gesprochen werden, und noch weniger sind solche Fälle auf Anpassung an Folgen geschlechtlicher Zuchtwahl zurickzuführen; wir haben es hier höchstens mit einer allgemeinen Anpassung zu tun, »einer Regenerationskraft des ganzen Organismus, die bis zu einem gewissen Grade überall in Tätigkeit treten kann, wo an dem betreffenden Tier etwas verloren geht, geschehe dies auch ganz ausnahmsweise. * Für diese Annahme spricht auch die hier besprochene, bis nun gar nicht bekannte Tatsache der Regeneration der Kiefer bei den Eidechsen, um so mehr als es nicht bekannt ist, daß dieselben im Freien in die Lage kämen, gewisser Kieferteile verlustig zu werden, und als diese Tiere im System den Vögeln ganz nahe stehen.

Um nicht einem etwaigen Einwande zu begegnen, daß den Eidechsen möglicherweise eine größere Regenerationskraft zukomme, als dies ihrer phyletischen Stellung nach zu erwarten wäre, unternahm ich es auch nachzuprüfen, ob die Eidechsen die Extremitäten oder wenigstens Teile derselben (Zehen) zu regenerieren vermögen. Aber meine diesbeztiglichen Versuche ergaben ein negatives Resultat. Gegen Mitte Juni ${ }^{2}$ ) amputierte ich an sieben sehr jungen Exemplaren die zweitunterste Zehe am rechten Hinterbein. Die Amputation ergab

1) 0. HüBNer kommt in einer Schrift über »Regeneration und ihre Beziehungen an Anpassungserscheinungen gelegentlich auf die Schnabelregeneration bei den Vögeln zu sprechen und spricht sich ganz im WeIsmansschen Sinne ans, indem er behauptet, daß die bekannten Fälle von der Schnabelregeneration als Anpassungserscheinungen aufzufassen sind, da >solche Fälle mit den Lebensgewohnheiten des Tieres und mit der Verlustfahigkeit des betreffenden Körperteils in enger Beziehung stehens. In diesem Sinne deutet er auch einen ihm bekannten Fall, wo eine Gans, welcher der halbe Oberschnabel fehlte, denselben nicht regenerierte. Trotzdem er selbst zugibt, daß er die Ursache des Defektes nicht mit Sicherheit angeben kann, zieht er doch den Schluß, daß eine Regeneration hier nicht möglich sei, da die Verletzung des Schnabels bei diesen Tieren ein seltenes Vorkommnis sei. Diese Ansicht kann nun keinesfalls als berechtigt angesehen werden, denn der angeführte Fall kann doch nicht als Beweis hierfür gelten, daß die Gans den Schnabel nicht regenerieren könne. Daß in diesem Falle die zu erwartende Regeneration des Oberschnabels ausblieb, ist wohl nur der Möglichkeit eines krankhaften Defektes oder irgendeinem andern ungünstigen Umstande, wie z. B. vorgeriicktes Alter des Tieres, zuzuschreiben, wodurch aber die Auffassung der Schnabelregeneration bei Vögeln als Anpassungserscheinung nicht im geringsten gerechtfertigt ist.

2. Da die Resultate dieses Versuchs negativ waren, so unterlasse ich es, das Versuchsjournal hieriiber anzuftihren. 
258 Isaak Werber, Regeneration der Kiefer bei der Eidechse Lacerta agilis.

eine sehr geringe Blutung, die Wunde schloß sich sehr rasch und war binnen wenigen Tagen ganz verheilt; der Stummel der amputierten Zehe wurde aber nicht regeneriert und ist bis jetzt (Mitte November) - also nach einem Zeitraum von 5 Monaten - unverändert geblieben. Es unterliegt wohl keinem Zweifel mehr, daß die Eidechsen die Extremitäten nicht zu regenerieren vermögen ${ }^{1}$ ).

\section{Literaturverzeichnis.}

EgGer, Ein Fall von Regeneration einer Extremität bei Reptilien. Arb. zool. Inst. Würzburg. Bd. 8. 7. 1888.

HüBNER, 0 , Neue Versuche aus dem Gebiete der Regeneration und ihre Beziehungen zu Anpassungserscheinungen. Fischer, Jena 1902.

Przibray, H., Die Regeneration bei den Crustaceen. Arb. Zool. Inst. Wien. XI. 1899. S. 163.

- Regeneration. Ergeb. der Physiol. 1. Jahrg. Bergmann, Wiesbaden 1902.

Weismans, Aug., Tatsachen und Auslegungen in Bezug auf Regeneration. Fischer, Jena 1899. S. 5.

— Vorträge über die Descendenztheorie. Fischer, Jena 1902.

Werner, F., Über die Schuppenbekleidung des regenerierten Schwanzes bei den Eidechsen. Sitz.-Ber. kais. Ak. d. Wissensch. 1896.

- Phylogenetische Studien über die Homologien und Veränderungen der Kopfschilder bei den Sehlangen. Arb. Zool. Inst. Wien. Tom XI. Heft 2. 1899.

WolfF, G., Entwickelungsphysiologische Studien. II. Archiv f. Entw.-Mech. Bd. XII. 1901. S. 349.

1) EGGER gibt zwar einen Fall an, wo eine Eidechse mit einer verstiimmelten Hinterextremität im Freien vorgefunden worden sein soll, die eine ganz abweichende Beschuppung anfwies, und ist geneigt, diesen Fall als Regeneration der Extremität anzusprechen. Dieser Fall ist aber eher auf eine Verletzung im embryonalen Zustande zurückzuführen, da es doch ganz ausgeschlossen ist, daß eine Eidechse eine ganze Extremität zu regenerieren imstande sei, wenn nicht einmal eine Zehe am ausgebildeten Tier regeneriert werden kann. 